\title{
Information/communication, deux pôles de nos études et de notre raison
}

Daniel Bougnoux

\section{(2) OpenEdition}

1 Journals

Édition électronique

URL : http://journals.openedition.org/communicationorganisation/1811

DOI : 10.4000/communicationorganisation. 1811

ISSN : $1775-3546$

Éditeur

Presses universitaires de Bordeaux

Édition imprimée

Date de publication : 1 novembre 1995

ISSN : 1168-5549

Référence électronique

Daniel Bougnoux, «Information/communication, deux pôles de nos études et de notre raison », Communication et organisation [En ligne], 8| 1995, mis en ligne le 26 mars 2012, consulté le 30 avril 2019. URL : http://journals.openedition.org/communicationorganisation/1811 ; DOI : 10.4000/ communicationorganisation. 1811

Ce document a été généré automatiquement le 30 avril 2019

(c) Presses universitaires de Bordeaux 


\title{
Information/communication, deux pôles de nos études et de notre raison
}

\author{
Daniel Bougnoux
}

1 Faut-il opposer communication à information? Il n'est pas facile de concevoir exactement leurs rapports, et de délimiter leurs domaines respectifs. Si l'information suppose en général la communication, comme sa base dont elle émerge, l'inverse n'est pas nécessaire : la communication ne conduit pas toujours à l'information, et s'en passe même assez bien. Dans un fou-rire, dans la chaleur communicative des affects, dans l'observation d'un rituel ou la participation aux règles d'une culture en général..., les hommes s'éprouvent reliés, ou membres d'une communauté, sans que cette conscience se rattache à un contenu cognitif (à une information) en particulier. Nous dirons, en hommage à une traditionnelle distinction des manuels de philosophie, que l'information vaut et se mesure dans le champ de la connaissance, et la communication dans celui de l'action ou de l'organisation. De ce partage se déduit que la seconde précède et conditionne la première.

2 Ce primat de la communication sur l'information peut s'établir d'une autre manière. En remarquant par exemple que le verbe communiquer se conjugue aisément à l'intransitif, comme si l'action ou l'opération ainsi désignées conservaient une forme secrètement réflexive (communiquer c'est mettre ou avoir en commun), voire impersonnelle : « entre nous, dit le couple, ça communique bien» (ou «on n'arrive pas à communiquer»). Le sujet de la communication est souvent indistinct, ou peu décidable. Le verbe informer en revanche exige un sujet et un complément d'objet nettement désignés : la grammaire de l'information est clairement secondaire alors que celle de la communication demeure primaire. Ce couple terminologique emprunté à la psychanalyse éclaire et renforce notre distinction; l'information donne lieu à des contenus, elle est soumise au principe de réalité, donc à l'alternative du vrai et du faux (on vérifie ou l'on réfute une information). Par contre quelle pourrait être la vérité d'une communication? Davantage aimantée par le principe de plaisir (d'être ensemble), la communication se moque en général de la vérité parce qu'elle réside en deçà, dans le tressage de la relation, le prolongement du 
contact ou l'organisation de la communauté. On ne demande pas aux liens en général d'être vrais, mais authentiques, chaleureux ou forts, valeurs assez différentes; et l'obligation faite à ceux qui passent dans les médias (au moins audio-visuels) « d'être soimême et d'avoir le contact » ne garantit nullement la vérité de leurs messages.

Notre distinction information/communication pourrait ainsi courir sur la même ligne que la distinction du contenu et de la relation, selon l'axiome posé par Watzlawick et alii à l'ouverture d'Une logique de la communication; ou que celle de l'énoncé et de l'énonciation. Énonciation, relation, communication: on vérifie dans chaque cas que celles-ci viennent d'abord, et qu'elles sont logiquement premières pour le calcul du sens (elles pilotent ou cadrent le contenu du message). La condition peut exister sans le conditionné, comme les jeux de la communication peuvent se passer de toute information. C'est le cas de la fonction phatique selon Jakobson, qu'il faudrait sans doute classer en tête des cinq autres si l'on s'intéressait à la dimension génétique et chronologique du célèbre tableau des six fonctions de la communication. De même que la relation peut se donner sans le contenu d'aucun message, ne peut-on concevoir l'énonciation sans énoncé correspondant (par exemple dans l'éclat de rire, l'interjection ou les usages infralinguistiques de la parole) ? Un autre trait remarquable, au plan de la description logique de ces trois notions, est qu'elles n'existent qu'au présent de l'acte, et ne se diffèrent pas (contrairement à leurs antonymes); de sorte que leur " vérité » (si l'on persiste à étendre à leur sphère cette notion) y est de type autoréférentiel : la preuve d'une relation c'est qu'on l'éprouve. Et celle d'une énonciation est autovalidante : j'ai beau proférer les pires bêtises, le fait que je les énonce se trouve automatiquement vrai.

Mais quittons le plan de cette description trop formelle pour celui des comportements. L'information et la communication, qui évoluent selon des logiques distinctes, engendrent des activités ou des métiers fort différents. Un enseignant n'est pas un animateur ; un journaliste ne se confond pas avec un publicitaire ou avec un dircom ; une enquête judiciaire ne se ramène pas à une transaction entre les parties, et un artiste digne de ce nom ne négocie pas le contenu de son œuvre ni ne met celle-ci aux voix... En écrivant mon petit livre La Communication contre l'information j'étais surtout soucieux de défendre cette émergence de l'information, ou la transcendance de formes hautes d'autorité symbolique (comme celles de l'École, de l'Église ou de l'État, pourvoyeuses d'identités ou de «bonnes formes »), qui ne peuvent sans danger se rabattre dans le jeu des relations publiques ni se galvauder dans des arrangements interpersonnels. Dans l'enseignement, dans l'investigation journalistique, dans la recherche scientifique, dans la justice ou dans l'art, pour citer quelques domaines donateurs d'informations fortes, la communication désigne un péril, celui des connivences ou des communautés instituées toujours prêtes à édulcorer l'appel du message, à émousser son tranchant dans la redondance des bonnes relations, dans le circuit éprouvé du lieu commun.

Dans le partage entre information et communication, la première apparaît donc comme une force d'ouverture et de progrès, et la seconde comme refermeture, tendance à la conservation des acquis et à la redondance communautaire. Mais à peine a-t-on insisté sur la contradiction possible entre nos deux termes qu'il faut corriger le schématisme, voire l'absurdité où peut conduire cette thèse, par l'affirmation de leur antagonisme complémentaire. L'information émerge de la communication mais peut aussi y demeurer captive; et toute société, par l'appareil médiatique dont elle se dote, demeure à la fois ouverte et fermée. 
6 J'en étais là de cette distinction quand sont apparues en France les grèves autour du «plan Juppé ». La mésaventure peu banale du Premier Ministre a mis en évidence plusieurs éléments qui touchent de près à notre distinction, et éclairent différemment ce qui précède. Le moins qu'on puisse conclure de la récente crise (loin d'être terminée à l'heure d'écrire ces lignes), c'est que l'art du gouvernement a des raisons que la raison ignore. Que penser de l'argument tenace, propagé par nos dirigeants et inégalement repris par la presse, selon lequel l'action d'Alain Juppé souffrirait d'un persistant "problème de communication»? Il semble que ceux qui mettent en avant ce terme défendent plus ou moins explicitement le schéma suivant : au poste panoramique qu'elles occupent nos élites disposent d'informations de grande qualité, traitées dans un cercle d'experts et de sages; malheureusement ces raisons et ces préoccupations des hommes d'en haut, porteurs et comptables par vocation de l'intérêt général (réduction des déficits, critères de convergence, échéancier européen...), échappent nécessairement aux hommes d'en bas (les masses, les syndicats et diverses corporations), empêtrés dans leurs intérêts catégoriels, dans la défense des privilèges acquis et du statu quo. Selon ce tranquille schéma la raison (politique, sociale) s'élabore au sommet, et il s'agit de la faire descendre dans les masses. C'est ce maillon somme toute subalterne de «la communication » d'un programme élaboré ailleurs qui aurait sauté dans la chaîne bien huilée des transmissions de la Raison d'État. D'où le mot d'ordre, répandu jusqu'au 7 décembre par l'entourage du Premier Ministre, d'avoir à expliquer sans relâche les mesures de "son " programme, mais sans rien négocier. « Vous n'avez pas compris... » répétait la main sur le cœur Alain Juppé avec la pointe d'agacement du professeur confronté à des cancres. « Négociations! » scandaient en chœur les manifestants.

7 Ce «malentendu » (dans quel sens ?) met en lumière les équivoques de ce qu'on appelle ordinairement communication ou raison politique. À commencer par la bifurcation radicale entre démocratie et technocratie: la démocratie repose sur des relations pragmatiques, c'est-à-dire horizontales ou en miroir, de sujets à sujets; la technocratie traite au contraire du politique ou du social comme d'un problème scientifico-technique, selon une relation descendante du sujet à l'objet. La notion même de Programme se classe comme approche technique et fait Pléonasme avec elle: programmer c'est écrire d'avance Une chaîne d'opérations, à exécuter séquentiellement point par point. Or le propre d'une relation de sujet à sujet en matière politique, psychologique ou sociale, c'est qu'on ne la programme pas. Une conversation, a fortiori une négociation ne peuvent s'écrire d'avance sans nier leur concept. Pourtant le rêve tenace ou l'erreur quasi Professionnelle des experts demeure de traiter les relations pragmatiques comme des relations techniques, en instrumentalisant la relation humaine. Le technocrate voudrait en finir avec la politique, cette irrationnelle vieillerie, il rêve par définition de "substituer au gouvernement des hommes l'administration des choses» (selon l'illustre prophétie du saint-simonisme). Mais les récents événements ont montré que cet oubli de la chose politique entraîne à de dangereux effets boomerang.

Quelle est plus précisément cette communication dont l'exigence se rappelle à chacun au cœur de la démocratie? La confusion entre négocier (relation pragmatique horizontale) et expliquer (relation pédagogique descendante), ou plutôt la substitution acharnée de la seconde à la première, a alimenté quelques semaines la récente crise. Au bout du compte il faut se mettre à table, c'est-à-dire accepter que la raison politique, loin de constituer le privilège des élites gouvernantes, soit peut-être « la chose du monde la mieux partagée ». Et que ce partage des vues et des voix fonde précisément notre démocratie. Quel 
renversement ! La « communication » du haut vers le bas est un cliché commode, et quasi naturel à qui parle depuis un lieu de pouvoir ou de discours fort (par exemple la chaire du professeur). Nous l'aura-t-on martelée, cette image du «premier de la classe » ou du «meilleur d'entre nous » qui colle à Alain Juppé! Mais celui qui a confondu la parole politique, par définition soumise à la ratification de ses destinataires, avec la parole magistrale du professeur sûr de ses contenus d'enseignements, en paye aujourd'hui le prix : les citoyens ne sont pas des écoliers, et la relation politique n'est pas vraiment pédagogique (l'école depuis 1968 ayant elle-même évolué vers moins d'arrogance).

Gouverner relève moins d'une science que d'un art, celui de faire croire, de faire désirer ou de faire vouloir; et l'alternative dans le discours politique n'est pas exactement celle du vrai et du faux, le ton du constat y demeure inapproprié ou sujet à suspicion. Si la chose même, cela qu'il faut obtenir ou produire s'appelle dans ce domaine la confiance, celle-ci échappera toujours à la parole constative et au rapport comptable : en parlant d'abord chiffres et grands équilibres (administration des choses), le Premier Ministre a commis, comme dirait Bateson, une erreur d'adresse ou de niveau logique. Il a oublié que gouverner ce n'est pas administrer mais, en effet, communiquer, et que communiquer c'est "entrer dans l'orchestre" (selon Bateson encore). Il serait intéressant de développer, contre le modèle d'une information descendante, quelques implications du modèle orchestral ou pragmatique en politique ; et de remarquer que le Premier Ministre s'est cramponné à sa partition, au risque de "jouer perso", et a gravement manqué d'oreille. Son appel incantatoire lors de sa prestation télévisée du 7 décembre à un monde commun («Quel beau Pays que la France!») ou à des soucis partagés (« Moi aussi je m'inquiète pour l'avenir de mes enfants...») est demeuré inaudible, tant l'inégalité des conditions dans la France de 1995 a morcelé le sentiment d'appartenir au même monde. Comment faire pour restaurer celui-ci? La correction des injustices les plus criantes dépasse largement un soi-disant " problème de communication ».

Sans nous étendre ici sur les motifs de ceux qui alléguaient haut et fort ce dernier, il reste à bien marquer dans la chose politique le primat absolu de la communication sur l'information, autrement dit la différence radicale entre l'enseignement et l'art du gouvernement. Les socialistes, largement issus des professions enseignantes, et imprégnés (au départ) d'une idéologie scientifique du changement social ont eux-mêmes connu quelques déboires dans leur communication du temps qu'ils étaient au pouvoir. En se conduisant à son tour comme un maître d'école le Premier Ministre a confondu quelques jours de trop la persuasion Politique avec la diffusion des connaissances. L'art du bon gouvernement ne se ramène pas à un problème information : non seulement Alain Juppé ne peut, de là où il parle et décide, tout savoir (les paramètres sociaux se mesurent mal et lui échappent relativement), mais quand bien même cette opacité constitutive du social se trouverait levée par une information idéale (inatteignable dans ce domaine), il ne pourrait au nom de celle-ci prétendre avoir raison tout seul.

11 L'étonnement d'Alain Juppé devant l'incompréhension, voire l'ingratitude des « masses », fait penser à celle d'un comique qui ne ferait rire personne mais persisterait à s'affirmer comique : ce n'est pas à lui de le dire, la preuve en incombe aux destinataires ou à la relation. Le problème serait différent avec un écrivain méconnu mais génial : celui-ci peut toujours, comme Stendhal, en appeler aux générations futures dans la mesure où l'information contenue dans ses livres par définition se diffère. Mais, nous l'avons déjà dit, l'énonciation ou la communication s'exercent en direct, au présent de la relation qui ratifie ou condamne avec très peu de marge d'appel; la communication comme la guerre 
est un art tout d'exécution. De sorte que l'homme politique "incompris » ne peut longtemps en accuser le peuple; et vouloir changer celui-ci, selon l'ironique conseil de Brecht, constituerait une nouvelle erreur de niveau logique. C'est lui qui a tout simplement faux, et c'est à lui de renouer d'urgence le contact, la relation ou comme on dit la confiance, qui constitue ici la chose même.

La vérité démocratique ou l'art de gouverner ne sont pas des branches ni des sousproduits d'une quelconque science. Fût-elle économique. La rationalité des affaires humaines (ta pragmata chez Aristote) est intrinsèquement limitée, comme l'ont établi Herbert Simon ou Michel Crozier, et il semble vain de rêver dans ce domaine d'un pilotage synoptique ou d'une information surplombante. Chacun traite les informations sociales et politiques selon les conditions de son monde propre, et la pluralité de ces mondes ne se laisse pas réduire à un monde commun, ou d'avance partagé. Qu'il était difficile aux cheminots, en voyant et en entendant Alain Juppé, de se projeter spontanément dans son monde ! Jacques Chirac, Charles Pasqua, voire les voix populistes, ont sur lui cette trouble supériorité de parler un langage affectif plus proche du peuple et de « savoir communiquer ».

Dans notre champ de l'inforcom, le paradoxe de la raison politique est ainsi de se mouvoir aux antipodes de la raison scientifique, enseignante ou technique. Dans quelques domaines de la culture une information autonome se laisse extraire, traiter par des individus isolés (éventuellement héroïques) et diffuser de haut en bas. L'information politique et sociale n'a pas cette complaisance. Fortement attachée à des mondes propre " et à des visions du monde (à des valeurs) irréductiblement conflictuelles, elle ne se totalise dans aucune parole singulière, ne se hiérarchise dans aucun grand dessein ni récit. Pour arriver dans ces domaines au moindre accord il ne faut pas se contenter d'expliquer, mais toujours remettre la raison sur le métier de la négociation. Car en démocratie la raison politique est communication; ou, pour paraphraser un poète, la politique « doit être faite par tous, non par un ».

\section{RÉSUMÉS}

Information et communication ne sont pas opposées mais complémentaires. La première, fondée sur le principe de raison, nécessite la seconde. Car la seconde se nourrit de la relation, de la «redondance communautaire", sans laquelle une information ne peut être partagée. Daniel Bougnoux s'appuie sur l'exemple du mouvement social de la fin de l'année 1995 pour analyser le «malentendu» entre le pouvoir et la nation. «Gouverner, ce n'est pas administrer mais (...) communiquer ». La communication précède l'information qui ne va jamais de soi.

Information and communication are not opposites but complementary. The former based on the principle of reason necessitates the latter, for this second one feeds off relations, from " community redondance", without which information cannot be shared. Daniel Bougnoux bases his work on the example of the social events at the end of 1995 in order to analyse the "misunderstanding » between the government and the nation. "Governing is not administrating but (...) communicating » Communication precedes information which never proceeds alone. 


\section{AUTEUR}

DANIEL BOUGNOUX

Daniel Bougnoux est ancien élève de l'École Normale Supérieure, agrégé de philosophie, et actuellement professeur de Sciences de la Communication à l'Université Stendhal de Grenoble 3. Il a publié à ce jour sept ouvrages, parmi lesquels La Communication par la bande (La Découverte, 1991, Prix Rhône Alpes du Livre), Sciences de l'Information et de la Communication (Larousse, collection « Textes essentiels ", 1993) et La Communication contre l'Information (Hachette, collection « Questions de Société », 1995). Son dernier livre est paru aux Éditions Arléa à la fin de mars 1996 : Lettre à Alain Juppé (et aux énarques qui nous gouvernent) sur un persistant " problème de communication ». Il prépare également l'édition en quatre volumes de l'œuvre romanesque d'Aragon dans la Bibliothèque de La Pléiade, dont le premier tome paraîtra en 1997. 